\title{
Fruit and vegetable intake of US adults estimated by two methods: What We Eat In America, National Health and Nutrition Examination Survey 2009-2012
}

\author{
M Katherine Hoy*, Joseph D Goldman and Rhonda S Sebastian \\ Food Surveys Research Group, Beltsville Human Nutrition Research Center, Agricultural Research Service, \\ US Department of Agriculture, 10300 Baltimore Avenue - Bldg 005, Beltsville, MD 20705, USA
}

Submitted 29 September 2015: Final revision received 7 February 2016: Accepted 1 March 2016: First published online 31 March 2016

\begin{abstract}
Objective: Estimates of fruit and vegetable (FV) consumption vary depending on intake definition, which may be determined by research purpose. Researchers have used two methods to evaluate intake: epidemiological and behavioural. The present study describes FV intake by adults using epidemiological $v$. behavioural approaches.

Design: One-day dietary intake data from What We Eat In America, National Health and Nutrition Examination Survey 2009-2012 were used. Sample weights were used to produce nationally representative estimates. FV intake (in cupequivalents (CE)) was estimated using the Food Patterns Equivalents Database. The epidemiological method considered all FV after disaggregating foods and beverages. The behavioural method included foods that provided at least $0 \cdot 2 \mathrm{CE}$ $\mathrm{FV}$ per $100 \mathrm{~g}$, and excluded sources high in fat, added sugar and $\mathrm{Na}$.

Setting: Nationally representative survey of the US population.

Subjects: Adults ( $n$ 10 563) aged $\geq 20$ years.

Results: For epidemiological $v$. behavioural, fruit intake was 1.1 v. 1.0 CE for males and 1.0 v. 0.9 CE for females. Vegetable intake was 1.8v.1.1 CE for males and 1.5 v. 1.0 CE for females.

Conclusions: The definition of FV intake affects estimates of consumption by the population and is an important consideration when planning and comparing research studies. The method used should align with research goals to assure accurate interpretation and validity of results.
\end{abstract}

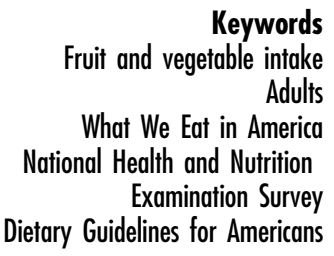

Recent efforts to promote consumption of fruit and vegetables (FV) have included the development of messages that are behaviourally focused. The Fruits \& Veggies - More Matters programme replaced the 5 A Day for Better Health Program in 2007 to reflect the recommendations in the 2005 Dietary Guidelines for Americans $(\mathrm{DGA})^{(1)}$. MyPlate is an icon developed to communicate recommendations of the 2010 DGA and now the 2015-2020 DGA ${ }^{(2)}$. FV are represented on half of the plate to convey their role in a meal relative to other foods. Formative research that contributed to the development of both programmes indicated that consumers preferred statements providing recommendations perceived to be easy to adopt and desired supplemental information focusing on actionable steps they could realistically take ${ }^{(1,3)}$. Accordingly, each message provides a foundation for sub-messages and programmes to support consumers in increasing $\mathrm{FV}$ intake. The primary indicator of both programmes' effectiveness will be an increase in FV intake.

FV may be consumed from foods in different forms. They may be eaten as discrete items and as integral parts of mixed dishes, such as stir-fried dishes and stews. In these latter types of food, $\mathrm{FV}$ are recognizable and their amounts are relatively substantial. In some foods, identifiable forms of FV are accompanied by high levels of energy from added sugar and fat, such as fruit desserts and fried potatoes. These are identified as nutrients of concern by the $\mathrm{DGA}^{(2)}$ because they are overconsumed. Although FV-containing foods with high amounts of added sugar and fat may contribute favourably to the intakes of other nutrients, such as $\mathrm{K}^{+}$and vitamin $\mathrm{C}$, they are generally not promoted as optimal choices. FV are also present in foods 
as additions, flavouring ingredients, or as components of items such as condiments. Amounts are small relative to other ingredients and the FV are often not visible. Intake of FV from these foods is largely incidental.

It has been noted that estimates of FV intake depend upon what foods are counted ${ }^{(4-6)}$. Cullen et $a l^{(4)}$ evaluated FV intake of seventy-one adolescents and fifty-four young adults using two approaches which they termed 'epidemiological' and 'behavioral'. An epidemiological method considers amounts provided by all food components, regardless of source or amount consumed. Thus, estimates include the contribution of some foods high in fat, added sugar and/or $\mathrm{Na}$. In the behavioural method, included foods contain $\mathrm{FV}$ in a specified minimum amount and items containing high amounts of overconsumed nutrients are not counted. The behavioural method resulted in estimates of $\mathrm{FV}$ intake up to $25 \%$ lower than the epidemiological method ${ }^{(4)}$.

The influence of using these different methods on estimates of $\mathrm{FV}$ intake of the adult population is not known. The purpose of the present study was to describe FV consumption in a nationally representative sample of US adults aged 20 years and older estimated using epidemiological and behavioural methods.

\section{Methods}

Estimates were based on $1 \mathrm{~d}$ of dietary data from 10563 adults aged 20 years and over (5380 females and 5183 males) who provided a complete $24 \mathrm{~h}$ recall in What We Eat in America (WWEIA), National Health and Nutrition Examination Survey (NHANES) 2009-2012. The NHANES sample was designed to be representative of the civilian, non-institutionalized US population, with oversampling of non-Hispanic blacks, non-Hispanic Asians (in 2011-2012 only), Hispanics, adults aged 80 years and older, and lowincome persons to improve the accuracy of estimates of health status indicators for these population subgroups ${ }^{(7)}$. Since the current study was a secondary analysis, no institutional review board approval was necessary.

\section{Dietary intake data collection and coding}

Dietary recall data were collected by trained interviewers using the US Department of Agriculture (USDA) Automated Multiple-Pass Method for the $24 \mathrm{~h}$ recall ${ }^{(8)}$. The current analysis used food intake data from the first of two non-consecutive dietary recall days, which were collected in person. All foods were coded using the USDA Food and Nutrient Database for Dietary Studies (FNDDS), which is the database of over 7000 foods, their nutrient values and weights for typical food portions used to process data from WWEIA, NHANES. Data reported in WWEIA, NHANES 2009-2012 were coded using FNDDS $5.0(2009-2010)^{(9)}$ and data in WWEIA, NHANES 2011-2012 were coded using FNDDS 2011-2012(10).

\section{Estimation of fruit and vegetable intake}

Estimates of the FV content of foods and beverages in FNDDS were obtained using the Food Patterns Equivalents Database (FPED) 2009-2010 ${ }^{(11)}$ and 2011-2012 $2^{(12)}$. The FPED converts the foods and beverages in FNDDS to thirty-seven USDA Food Patterns components. Food Patterns provide energy-based guidance on how much should be consumed from five main components - Fruit, Vegetables, Grains, Protein Foods, Oils - and their subcomponents, while limiting the amounts of added sugars, solid fats and alcoholic beverages. FV are expressed as cup-equivalents (CE) per $100 \mathrm{~g}$. Single-component foods such as $100 \%$ orange juice and carrots can be converted directly to FV Food Pattern components. Multi-ingredient foods, such as mixed dishes, condiments and some beverages, are disaggregated into ingredients and any FV contained in the food is assigned to its respective Food Pattern component(s). For example, the tomato in ketchup is counted towards total vegetable intake and any orange juice in an orange fruit drink is included in estimates of fruit intake. Details about the FPED methodology can found at online (http://www.ars.usda.gov/Services/docs. htm?docid=23871).

In the epidemiological method, the CE amounts of FV consumed from all reported foods and beverages were totalled, regardless of source or amount provided. This included any amount of FV contained in items such as condiments, bakery goods and beverages.

In the behavioural approach, intake of $\mathrm{FV}$ was considered for inclusion if it was in an identifiable form as either a discrete item (e.g. apple, broccoli, 100\% fruit or vegetable juice) or an integral part of a mixed dish (e.g. stew or stir-fry). Intake from mixed dishes was counted if it contained approximately $1 / 2$ cup FV per 1 cup serving. In FPED, this amount equated to approximately $0 \cdot 2 \mathrm{CE}$ per $100 \mathrm{~g}$ of a mixed dish, which is congruent with the MyPlate message for $\mathrm{FV}$ and the criteria used by Cullen et $a l^{(4)}$. Likewise, it is consistent with Food and Drug Administration criteria for health claims on labels of mixed dishes ${ }^{(13)}$, which are also guidelines of the Fruits \& Veggies - More Matters programme for licensing use of the brand $\operatorname{logo}{ }^{(14)}$. FV were not counted towards intake if the food item is not generally considered an optimal way to meet recommendations (e.g. fried potatoes, desserts, alcoholic mixed drinks) or if present in minor amounts (e.g. fruit drinks, condiments), which are measured by FPED. Thus, foods included in estimates using the behavioural approach were a subset of those using the epidemiological method.

\section{Statistical analyses}

To describe the dietary intake of FV using each method, the mean intake in $\mathrm{CE}$ and percentage reporting any intake were calculated. Analyses were carried out using the statistical software package SAS $^{\circledR}$ release $9 \cdot 3$ (2011). SUDAAN release $11 \cdot 0$ (2012) was used to adjust for survey 
design effects resulting from NHANES' complex, multistage probability sampling. All analyses used sample weights to produce nationally representative estimates.

\section{Results}

Estimates of FV intake using both approaches are shown in Table 1. Using the epidemiological method, the mean estimate of fruit intake for both males and females was $0 \cdot 1 \mathrm{CE}$ higher than the behavioural estimate. Mean intake of vegetables estimated by the epidemiological method was 0.7 and $0.5 \mathrm{CE}$ higher than the behavioural estimate for males and females, respectively.

Table 2 shows that $68 \%$ of males and $76 \%$ of females reported any intake of fruit on a day using the epidemiological approach $v .52 \%$ and $59 \%$, respectively, using the behavioural method. For vegetables, the percentage of adults reporting any intake on a day was approximately $95 \%$ by the epidemiological approach, and $70 \%$ and $73 \%$ for males and females, respectively, using the behavioural method.

\section{Discussion}

The definition of FV intake - that is, what foods/beverages are considered as contributing to intake - can substantially affect estimates of consumption. Compared with the epidemiological method, estimates using the behavioural approach were almost $10 \%$ lower for fruit and up to $25 \%$ lower for vegetables. Also, about $17 \%$ and $25 \%$ fewer individuals, respectively, reported any fruit or any vegetable intake by the behavioural approach.

These results may have important implications for evaluating behavioural messages and programmes, and for comparing and interpreting research about relationships between FV and health. In epidemiology, including intake of FV components from all foods is important for estimating nutrient intakes of the population and studying diet-health associations. Omitting some foods, such as fried potatoes, may result in underestimating nutrient consumption and obscure potential relationships. For instance, white potatoes contribute about $6 \%$ to both $\mathrm{K}^{+(15)}$ and fibre ${ }^{(16)}$ intakes; half of potato consumption is fried potatoes ${ }^{(15)}$. Small amounts of hidden ingredients in mixed dishes and condiments that may be excluded by the behavioural approach, such as onions, peppers and celery, were found to be important contributors to intake of polyphenolic compounds associated with health benefits ${ }^{(17)}$.

However, a behavioural approach is more relevant for evaluating consumer behaviour and effectiveness of interventions and messages related to FV consumption. In addition to increasing FV intake, the DGA recommends making shifts from more 'typical' choices, such as fried potatoes, to 'nutrient dense' foods and beverages lower in fat and added sugar as well as $\mathrm{Na}^{(2)}$. For most individuals,

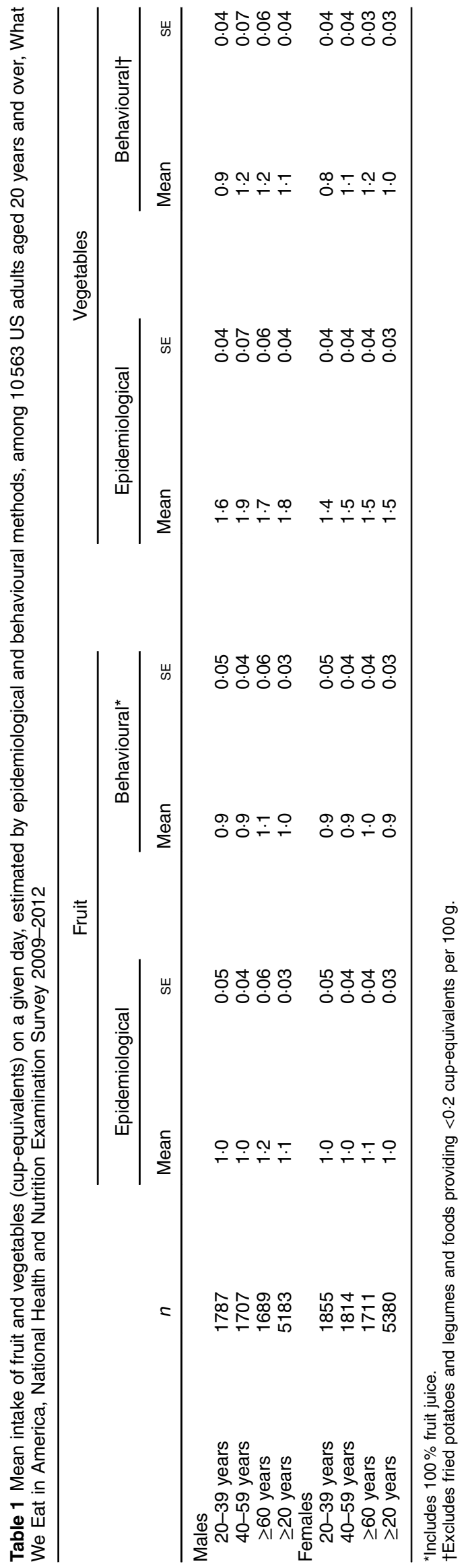


applying these guidelines often requires conscious effort. The behavioural approach can provide information about the actions consumers take to make choices consistent with the DGA, such as choosing healthier versions of $\mathrm{FV}$, including FV more frequently at meals and snacks, and selecting FV options at restaurants and convenience stores. Evaluating intake using both methods together can provide additional information about consumer choices and how foods contribute to FV intake.

The present study has several limitations. Evaluating FV intake using a behavioural approach requires an operational definition of intake. In the present study, the behavioural approach assumed that when FV were present in substantial quantities and were identifiable, the individual consciously chose to eat them $v$. not consuming them. It also presumed that intake from trivial amounts of FV in foods was not considered by the individual to count. Several observations provide some limited support for these criteria. Lettuce and tomato added to sandwiches were generally considered to count towards vegetable intake ${ }^{(18)}$, and they were counted towards intake in the present study since they can be deliberately added or removed. Most individuals knew ketchup contains a vegetable, and grape jelly has fruit ${ }^{(19)}$, but they did not necessarily count these items as intake ${ }^{(18)}$. However, although not optimal, some individuals also counted the visible fruit in fruit pies towards fruit intake, suggesting that since fruit was identifiable, individuals were aware of consuming it and considered it to count towards intake ${ }^{(19)}$. This may also apply to selection of other identifiable forms of $\mathrm{FV}$, such as fried potatoes.

Another problem with assigning behavioural criteria for defining FV intake is that knowledge and perceptions about FV vary widely and may be influenced by culture and ethnicity ${ }^{(18,19)}$. For instance, in cognitive testing of respondents from different backgrounds ${ }^{(19)}$, agreement was mixed that tomatoes, potatoes and particularly black beans were vegetables, whereas there was general concurrence regarding classification of corn and peppers as such. Rice was classified as a vegetable by about $20 \%$ of individuals, especially Spanish speakers ${ }^{(18)}$. In contrast to most English-speaking individuals, less than half of nonEnglish speaking people considered beef stew to provide vegetables ${ }^{(19)}$, a difference that might be explained by lack of familiarity with the dish or cultural differences in recipes. For some, tomato sauce in mixed dishes was not considered a vegetable ${ }^{(18)}$. In the present study, it was counted towards intake when it was added to a food, such as spaghetti, or when a mixed dish met the inclusion criterion of $0.2 \mathrm{CE}$ per $100 \mathrm{~g}$. More research is needed to understand consumer knowledge and perceptions about FV (especially among individuals from different cultures and backgrounds), and education is needed about healthful choices that count towards intake.

Lastly, estimates are based on $1 \mathrm{~d}$ of self-reported dietary data, which does not represent the variability in usual intakes by individuals. However, $1 \mathrm{~d}$ of dietary data 
from a nationally representative sample like WWEIA, NHANES adequately estimates mean intakes of the population when appropriate sample weights are applied to account for survey non-response and to balance the collection of $24 \mathrm{~h}$ recalls across days of the week ${ }^{(8)}$.

\section{Conclusion}

In conclusion, two methods for evaluating FV intake of adults produced substantially different estimates of the FV intake of US adults, particularly for vegetables. These results illustrate the magnitude of differences that can result from various definitions. Numerical estimates of intake are important for nutrition monitoring, programme evaluation and research; use of consistent methods is important in these activities. Evaluation of FV intake can provide information about consumer behaviour and help identify future needs for messages and interventions promoting healthful FV choices. There are various ways of defining intake and the method used should align with research goals to enhance interpretation and validity of results.

\section{Acknowledgements}

Financial support: No external financial support or funding was obtained, with the exception of salaries of the authors. Conflict of interest: The authors have no conflict of interest to disclose. Authorship: M.K.H. conceived and planned the research, and drafted and edited the manuscript. J.D.G. conducted all analyses of data for the study, and reviewed and edited the manuscript. R.S.S. contributed to planning the research and to writing and editing the manuscript. Ethics of buman subject participation: This study was conducted according to the guidelines laid down in the Declaration of Helsinki and all procedures involving human subjects were approved by the NCHS Research Ethics Review Board. Written informed consent was obtained from all subjects.

\section{References}

1. Pivonka E, Seymour J, McKenna J et al. (2011) Development of the behaviorally focused Fruits and Veggies - More Matters public health initiative. J Am Diet Assoc 111, 1570-1577.

2. US Department of Agriculture \& US Department of Health and Human Services (2016) Dietary Guidelines for Americans, 2015-2020, 8th ed. Washington, DC: US Government Printing Office.

3. US Department of Agriculture, Center for Nutrition Policy and Promotion (2011) Development of 2010 Dietary Guidelines for Americans Consumer Messages and New Food Icon: Executive Summary of Formative Research. http://www. choosemyplate.gov/sites/default/files/printablematerials/ ExecutiveSummaryOfFormativeResearch.pdf (accessed December 2014).

4. Cullen KW, Baranowski T, Baranowski J et al. (1999) Behavioral or epidemiologic coding of fruit and vegetable consumption from 24-hour dietary recalls: research question guides choice. J Am Diet Assoc 99, 849-851.
5. Eldridge AL, Smith-Warner SA, Lytle LA et al. (1998) Comparison of 3 methods for counting fruits and vegetables for fourth-grade students in the Minnesota 5 A Day Power Plus Program. J Am Diet Assoc 98, 777-782.

6. Roarke RA \& Niederhauser VP (2013) Fruit and vegetable intake: issues with definition and measurement. Public Health Nutr 16, 2-7.

7. Johnson CL, Dohrmann SM, Burt VL et al. (2014) National Health and Nutrition Examination Survey: Sample Design, 2011-2014. Vital and Health Statistics, series 2, no. 162. Hyattsville, MD: US Department of Health and Human Services, Centers for Disease Control and Prevention, National Center for Health Statistics.

8. Moshfegh AJ, Rhodes DG, Baer DJ et al. (2008) The US Department of Agriculture Automated Multiple-Pass Method reduces bias in the collection of energy intakes. Am J Clin Nutr 88, 324-332.

9. US Department of Agriculture, Agricultural Research Service (2012) USDA Food and Nutrient Database for Dietary Studies, 5.0 - Documentation and User Guide. Beltsville, MD: Food Surveys Research Group; available at http://www.ars. usda.gov/SP2UserFiles/Place/80400530/pdf/fndds/fndds5_ doc.pdf

10. US Department of Agriculture, Agricultural Research Service (2014) USDA Food and Nutrient Database for Dietary Studies 2011-2012: Documentation and User Guide. Beltsville, MD: Food Surveys Research Group; available at http://www.ars.usda.gov/SP2UserFiles/Place/80400530/ pdf/fndds/fndds_2011_2012_doc.pdf

11. Bowman SA, Clemens JC, Thoerig RC et al. (2013) Food Patterns Equivalents Database 2009-10: Methodology and User Guide. Beltsville, MD: Food Surveys Research Group; available at http://www.ars.usda.gov/SP2UserFiles/Place/ 80400530/pdf/fped/FPED_0910.pdf

12. Bowman SA, Clemens JC, Friday JE et al. (2014) Food Patterns Equivalents Database 2011-12: Methodology and User Guide. Beltsville, MD: Food Surveys Research Group; available at http://www.ars.usda.gov/SP2UserFiles/Place/ 80400530/pdf/fped/FPED_1112.pdf

13. US Food and Drug Administration, Center for Food Safety and Applied Nutrition (2008) Code of Federal Regulations Title 21 - Food and Drugs, Section 101.13. http://www. accessdata.fda.gov/scripts/cdrh/cfdocs/cfcfr/CFRSearch.cfm (accessed January 2015).

14. Produce for Better Health Foundation (2006) Fruits \& Veggies - More Matters. Products Promotable and Recipe Criteria. http://pbhfoundation.org/licensing/guid/ (accessed December 2014).

15. Hoy MK \& Goldman JD (2012) Potassium intake of the US population: What We Eat in America, NHANES 2009-2010. Food Surveys Research Group Dietary Data Brief no. 10. http://ars.usda.gov/Services/docs.htm?docid=19476 (accessed January 2016).

16. Hoy MK \& Goldman JD (2014) Fiber intake of the US population: What We Eat in America, NHANES 2009-2010. Food Surveys Research Group Dietary Data Brief no. 12. http://ars. usda.gov/Services/docs.htm?docid=19476 (accessed January 2016).

17. Sebastian RS, Wilkinson Enns C, Goldman JD et al. (2015) A new database facilitates characterization of flavonoid intake, sources, and positive associations with diet quality among US adults. J Nutr 145, 1239-1248.

18. Wolfe WS, Frongillo EA \& Cassano PA (2001) Evaluating brief measures of fruit and vegetable consumption frequency and variety: cognition, interpretation, and other measurement issues. J Am Diet Assoc 101, 311-318.

19. Thompson FE, Willis GB, Thompson OM et al. (2011) The meaning of 'fruits' and 'vegetables'. Public Health Nutr 14, $1222-1228$. 